\title{
VECTOR OPTIMIZATION PROBLEMS WITH LINEAR CRITERIA OVER A FUZZY COMBINATORIAL SET OF ALTERNATIVES
}

\author{
N. V. Semenova, ${ }^{a}$ L. N. Kolechkina, ${ }^{b}$ and A. M. Nagirna ${ }^{c}$
}

UDC 519.85

\begin{abstract}
Vector optimization problems over a fuzzy combinatorial set of permutations are investigated. Based on the properties of the convex hull of a fuzzy combinatorial set of permutations, modifications of multicriteria choice methods are developed and substantiated for a fuzzy feasible combinatorial set. Mathematical models of some application problems are presented.
\end{abstract}

Keywords: multicriteria optimization, discrete optimization, fuzzy set, membership function, combinatorial set of permutations, Pareto optimal solutions, fuzzy set of alternatives, fuzzy multiset.

\section{INTRODUCTION}

In real situations of decision making, objectives, constraints, and choice criteria are generally subjective and are not precisely defined. Therefore, modeling of decision making needs fuzzy logic and fuzzy sets and relations. Fuzzy concepts allow modeling smooth, gradual change of properties and unknown functional dependences expressed as qualitative constraints.

In many applied problems, initial data cannot often be specified exactly. Such situations reflect the lack of information in problem statement since decision making under fuzzy conditions and criteria appears problematic. In modeling real problems, fuzziness is manifested in the description of functions and parameters on which they depend. The fuzzy set theory proposed by Zadeh in [1] is a convenient mathematical tool to describe and take into account such information. Fuzzy sets are widely used in various applications of artificial intelligence, pattern recognition, decision theory, etc.

In many theoretical and practical problems of operations research and complex systems engineering, the need arises to make decisions that meet several optimality criteria. At the same time, multicriteria choice problems with a set of alternatives that can be estimated both quantitatively and qualitatively are quite popular. Under multicriteria conditions, the most reasonable decision is chosen from a set of unimprovable alternatives. The problem of searching for such a set is of large practical and theoretical importance. Moreover, the cardinal number of the set of alternatives can be very large in real problems, which makes the decision-making problem rather complex. The book [2] presents a mathematical statement and axiomatic substantiation of the Edgeworth-Pareto principle (known since the 19th century) for the case of exact preference relation of decision-maker. This principle is a guideline in choosing best decisions in economics and engineering when several objective functions (criteria) should be taken into account simultaneously. However, it is not universal and is true only in solving a certain yet rather wide class of multicriteria choice problems. Applying it beyond this class is risky or inadmissible.

We will extend the Edgeworth-Pareto principle to a wider class of multicriteria problems with a fuzzy set of feasible solutions.

V. L. Volkovich, I. V. Sergienko, V. A. Perepelitsa, Yu. Yu. Chervak, R. Kini, H. Raiffa, E. S. Ventzel', Yu. B. Germeyer, et al. made a significant contribution to the development of multicriteria optimization methods and their application in problems of control and design of complex systems. The studies [2-9] deal with various aspects of the vector

${ }^{a}$ V. M. Glushkov Institute of Cybernetics, National Academy of Sciences of Ukraine, Kyiv, Ukraine, nvsemenova@meta.ua. ${ }^{b}$ Poltava University of Economics and Trade, ludapl@ukr.net. ${ }^{c}$ Ukrainian State University of Finance and International Trade, Kyiv, Ukraine, vpn2006@rambler.ru. Translated from Kibernetika i Sistemnyi Analiz, No. 2, pp. 88-99 March-April 2011. Original article submitted July 8, 2009. 
optimization theory. I. V. Sergienko, V. S. Mikhalevich, N. Z. Shor, et al. played an important role in the development of new methods to solve combinatorial discrete optimization problems. The methods they created are widely applied in solving many problems of scheduling theory, manufacture location, and network and gas pipeline design.

In practice, unfuzzy optimization theory is often applied to fuzzy models where there are no grounds to specify coefficients as exact numbers. Such an artificial contraction of the a priori information may distort the final results. Problems that take into account fuzzy boundaries in both objective functions and constraints specified on combinatorial sets are of current importance. The fuzzy choice problem is a direct generalization of the ordinary (exact) choice problem, its analysis is of doubtless theoretical and practical interest, and its solutions (the fuzzy set of chosen decisions) can form the basis for the subsequent final choice. Analyzing problem is also useful from the point of view of its applications in various fields.

The books [10-12] consider multicriteria problems under fuzzy information about the objective functions and feasible domain, and [13-16] deal with problems on combinatorial sets. It is expedient to consider a problem that combines these problems.

In what follows, we will define a vector optimization problem over a combinatorial set of permutations and take into account the fact that the convex hull of such a set is a general permutable polyhedron whose set of vertices composes the considered combinatorial set $[14,15]$. The investigated properties of this set allow reducing the solution of the problem defined on a discrete combinatorial set to a problem with continuous feasible domain. We will analyze the multicriteria problem in view of the combinatorial properties of the fuzzy set of feasible alternatives and will propose approaches to solve it.

\section{PRELIMINARY INFORMATION. BASIC CONCEPTS AND DEFINITIONS}

A fuzzy subset is formed by introducing the generalized concept of membership, i.e., extension of the two-element set $\{0,1\}$ of the characteristic function to the continuum $[0,1]$. This means that the passage from the full membership of an object to the full nonmembership occurs smoothly, the membership may be expressed by any number from the unit interval $[0,1]$, not by only one of the two values of elements of the set $\{0,1\}$, as in the case of indicators of ordinary subsets. This property of fuzzy subsets allows a set-theoretic representation of real fuzzy concepts. The possibility of formalizing concepts turned out to be quite useful, for example, for the development of principles of computer artificial intelligence that model human thinking processes.

Irrespective of whether fuzzy or exact subsets are used, determining the degrees of membership leans upon some subjective criteria of the decision maker (DM). However, while one of two numbers (zero or unity) if chosen to determine exact subsets, the variety of membership degrees to determine fuzzy subsets is much greater. In some cases, determining appropriate membership degrees of elements of fuzzy sets leads to severe difficulties in dealing with fuzzy concepts.

Generally, a fuzzy mathematical programming problem can formally be described as follows [11]. Let $X$ be a universal set of alternatives and $\mu_{A}: X \rightarrow[0,1]$ be a given fuzzy subset of feasible alternatives; $Y$ be a universal set of estimates of the results of choosing alternatives from the set $X$, and $\mu_{R}: Y \times Y \rightarrow[0,1]$ be a fuzzy preference relation specified on the set $Y$. Alternative decisions are estimated by fuzzy values of the given fuzzy objective function $\varphi: X \times Y \rightarrow[0,1]$. The task is to rationally choose alternatives based on the information specified in the described form.

The next step toward improving the model is to describe the parameters as fuzzy subsets. Along with specifying the set of feasible values of the parameters, additional information in terms of functions of membership in these sets is introduced in the model. These membership functions are considered as a way for an expert to approximately represent, in aggregated form, the available unformalized concept of the real value of the parameter. The value of the membership function can be understood as weight coefficients assigned by the expert to different possible values of the parameter.

Considering such additional information complicates the original mathematical model; nevertheless, it may appear simpler than the model that takes into account the variety of additional factors.

For the further presentation, let us define the generalizations of the concepts of a multiset, $n$-sample, and a combinatorial set of permutations to the case of fuzzy information.

Definition 1. A fuzzy multiset $\widetilde{X}$ over a universal set $X$ is a collection of pairs $\left(x, \mu_{\tilde{X}}(x)\right)$, where $x \in X$, and $\mu_{\tilde{X}}(x)$ is a function $x \rightarrow[0,1]$ called membership function of the multiset $\tilde{X}$. The value of $\mu_{\tilde{X}}(x)$ for a specific $x$ is called the degree of the membership of this element in the fuzzy multiset $\tilde{X}$. 
Let us recall that by definition, multisets form a subclass of fuzzy multisets. A number of operations such as union, intersection, Cartesian product, difference, etc. can be performed on fuzzy sets just as on ordinary sets. The same operations hold for fuzzy multisets [16].

Let a fuzzy multiset

$$
\widetilde{A}=\left\{a_{1}, \mu_{\widetilde{A}}\left(a_{1}\right), a_{2}, \mu_{\widetilde{A}}\left(a_{2}\right), \ldots, a_{q}, \mu_{\widetilde{A}}\left(a_{q}\right)\right\}
$$

be given, its support set be

$$
S(\widetilde{A})=\left\{e_{1}, \mu_{\widetilde{A}}\left(e_{1}\right), e_{2}, \mu_{\widetilde{A}}\left(e_{2}\right), \ldots, e_{k}, \mu_{\widetilde{A}}\left(e_{k}\right)\right\},
$$

where

$$
\mu_{\widetilde{A}}\left(e_{i}\right)=\min \left\{\mu_{\widetilde{A}}\left(a_{i_{j}}\right) \mid a_{i_{j}}=a_{i_{t}}, j \neq t, \forall i, j, t \in N_{q}\right\}, e_{j} \in R \quad \forall j \in N_{k}=\{1, \ldots, k\},
$$

and the multiplicity of elements $k\left(e_{j}\right)=r_{j}, \quad j \in N_{k}, r_{1}+r_{2}+\ldots+r_{k}=q$.

An ordered fuzzy $n$-sample out of a fuzzy multiset $\tilde{A}$ is a set

$$
a=\left(a_{i_{1}}, \mu_{\widetilde{A}}\left(a_{i_{1}}\right), a_{i_{2}}, \mu_{\widetilde{A}}\left(a_{i_{2}}\right), \ldots, a_{i_{n}}, \mu_{\widetilde{A}}\left(a_{i_{n}}\right)\right),
$$

where $\left(a_{i_{j}}, \mu_{\widetilde{A}}\left(a_{i_{j}}\right)\right) \in \tilde{A} \forall i_{j} \in N_{k}, \forall j \in N_{k}, i_{s} \neq i_{t}$, if $s \neq t \quad \forall s \in N_{k}, \forall t \in N_{k}$.

Definition 2. A fuzzy set $P(\widetilde{A})$, whose elements are fuzzy $n$-samples (1) out of a fuzzy multiset $\widetilde{A}$ is called a fuzzy Euclidean combinatorial set if the conditions $\left(a^{\prime} \neq a^{\prime \prime}\right) \Leftrightarrow\left(\exists j \in N_{n}:\left(a_{j}^{\prime}, \mu_{\widetilde{A}}\left(a_{j}^{\prime}\right)\right) \neq\left(a_{j}^{\prime \prime}, \mu_{\widetilde{A}}\left(a_{j}^{\prime \prime}\right)\right)\right.$ are satisfied for arbitrary pair of its elements

$$
\begin{aligned}
& a^{\prime}=\left(a_{1}^{\prime}, \mu_{\widetilde{A}}\left(a_{1}^{\prime}\right), a_{2}^{\prime}, \mu_{\widetilde{A}}\left(a_{2}^{\prime}\right), \ldots, a_{n}^{\prime}, \mu_{\widetilde{A}}\left(a_{n}^{\prime}\right)\right), \\
& a^{\prime \prime}=\left(a_{1}^{\prime \prime}, \mu_{\widetilde{A}}\left(a_{1}^{\prime \prime}\right), a_{2}^{\prime \prime}, \mu_{\widetilde{A}}\left(a_{2}^{\prime \prime}\right), \ldots, a_{n}^{\prime \prime}, \mu_{\widetilde{A}}\left(a_{n}^{\prime \prime}\right)\right)
\end{aligned}
$$

i.e., the set $P(\tilde{A})$ has the following property: two elements of the set $P(\tilde{A})$ are different if irrespective of other differences they differ in the order of symbols constituting them and in the degree of membership to the fuzzy set $P(\widetilde{A})$.

A fuzzy set of permutations with repetitions of $n$ real numbers among which $k$ are different is called general fuzzy set of permutations and is denoted by $P_{n k}(\widetilde{A})$.

Definition 3. A convex combination of fuzzy sets $A_{1}, A_{2}, \ldots, A_{n}$ in $R^{n}$ is a fuzzy set $A$ with the membership function

$$
\mu_{A}(x)=\sum_{i=1}^{n} \lambda_{i} \mu_{i}(x)
$$

where $\lambda_{i} \geq 0, i \in N_{n}, \sum_{i=1}^{n} \lambda_{i}=1$.

We will consider elements of a fuzzy set of permutations with repetition as points of the arithmetic Euclidean space $R^{n}$.

Without loss of generality, we order elements of the multiset $\tilde{A}$ as follows:

$$
a_{1} \leq a_{2} \leq \ldots \leq a_{n} .
$$

Along with the classical permutation polyhedron introduced by Rado [14], let us describe a general permutation polyhedron $\Pi_{n k}(\tilde{A})$, which is the convex hull of the general set of permutations $P_{n k}(\tilde{A})$ and can be described by a system of linear inequalities [15]:

$$
\begin{gathered}
\sum_{j=1}^{n} x_{j} \leq \sum_{j=1}^{n} a_{j}, \sum_{j=1}^{i} x_{\alpha_{j}} \geq \sum_{j=1}^{i} a_{j}, \\
\alpha_{j} \in N_{n}, \alpha_{j} \neq \alpha_{t}, \forall j \neq t, \forall j, t \in N_{i}, \forall i \in N_{n}, P_{n k}(A)=\operatorname{vert} \Pi_{n k}(A),
\end{gathered}
$$

where vert $\Pi_{n k}(A)$ is the set of vertices of the polyhedron $\Pi_{n k}(A)$.

The fuzzy convex polyhedron $\Pi_{n k}(\tilde{A})$ can also be represented as the convex hull of a fuzzy combinatorial set of permutations: $\Pi_{n k}(\widetilde{A})=\operatorname{conv} P_{n k}(\tilde{A})$. 
As a rule, by a multicriteria optimization problem is meant minimizing or maximizing a vector criterion over a feasible set of alternatives. The main properties of alternatives such as value, utility, cost, etc. can be represented formally using a vector objective function. The fuzziness of the statement of a multicriteria optimization problem can be in the descriptions of both the set of alternatives and criteria functions. Different forms of the description of the initial information lead to different statements of fuzzy optimization problems: a problem of attaining a fuzzy objective under fuzzy constraints; a fuzzy optimization problem under a fuzzy set of feasible alternatives; a fuzzy version of a standard optimization problem with "relaxed" criteria and/or constraints (i.e., instead of an optimization problem, a problem of satisfying the objective is solved and the inequalities for the objective function and constraints may be violated); an optimization problem with fuzzy coefficients, etc.

In the present paper, the task is to maximize a vector function $F$ on a fuzzy Euclidean combinatorial set $\tilde{X}$.

Consider a combinatorial multicriteria optimization problem of the form

$$
Z(F, X): \max \left\{F(x) \mid x \in X \subset R^{n}\right\}, F(x)=\left(f_{1}(x), \ldots, f_{l}(x)\right), f_{i}: R^{n} \rightarrow R, i \in N_{l},
$$

where $X=$ vert $\Pi_{n k}(A) \cap D \neq \varnothing, \Pi_{n k}(A)=\operatorname{conv} P_{n k}(A), P_{n k}(A)$ is a combinatorial set of permutations, and $D \subset R^{n}$ is a convex polyhedron.

A fuzzy subset $\tilde{X}=\left\{x, \mu_{\tilde{X}}(x)\right\}$, where $x \in X$, is specified on the set $X, \mu_{\tilde{X}}(x): X \rightarrow[0,1]$ is the function of membership in the set $\tilde{X}$, which is called a fuzzy set of alternatives. By maximization we will mean choosing a fuzzy subset $R$ from the fuzzy set $\widetilde{X}$ to which there corresponds the largest value of both the vector function $F$ and the membership function $\mu_{\tilde{X}}(x)$ of the fuzzy set of alternatives. Depending on the way they are compared, these alternatives in multicriteria optimization problems are called efficient (Pareto optimal), weakly efficient (in the sense of Slater), and strictly efficient (in the sense of Smale) and are denoted, respectively, by $P(F, \widetilde{X}), \operatorname{Sl}(F, \tilde{X})$, and $\operatorname{Sm}(F, \tilde{X})$.

Definition 4. An alternative $x^{*} \in \tilde{X}$ is called efficient if there is no another alternative $x \in \tilde{X}$ such that $F(x) \geq F\left(x^{*}\right)$, $\mu_{D}(x) \geq \mu_{D}\left(x^{*}\right)$ and at least one inequality is strict; weakly efficient if $\bar{\exists} x \in \tilde{X}: F(x)>F\left(x^{*}\right), \mu_{D}(x)>\mu_{D}\left(x^{*}\right)$, and strictly efficient if $\bar{\exists} x \in \tilde{X}: x \neq x^{*}, F(x) \geq F\left(x^{*}\right), \mu_{D}(x) \geq \mu_{D}\left(x^{*}\right)$.

As follows from the definitions, $\operatorname{Sm}(F, \tilde{X}) \subset P(F, \tilde{X}) \subset \operatorname{Sl}(F, \tilde{X})$.

Represent the original problem $Z(F, X)$ as an $(l+1)$-criteria problem

$$
F(x) \rightarrow \max , \mu_{\tilde{X}}(x) \rightarrow \max , x \in \tilde{X} .
$$

By the solution of a problem with a fuzzy set of alternatives we will mean a fuzzy set with the membership function

$$
\mu(x)=\left\{\mu_{\tilde{X}}(x)|x \in P(F, \tilde{X}) \vee 0| x \notin P(F, \tilde{X})\right\} .
$$

Thus, the fuzzy set of solutions includes those and only those alternatives of the universal set $X$ that produce the values of the vector function $F(x)$ and membership function $\mu_{\tilde{X}}(x), x \in \tilde{X}$, unimprovable simultaneously.

Let $\tilde{Y}=\left\{y \in R^{l} \mid y=F(x), x \in \tilde{X}\right\}$ be the set of attainable vector estimates that are specified by the fuzzy values of vector estimates $y=\left(y_{1}, y_{2}, \ldots, y_{l}\right)$, and $P(\alpha)$ be the set of all efficient alternatives of the $(l+1)$-criteria problem

$$
y_{i} \rightarrow \max , i \in N_{l}, \mu_{D}(x) \rightarrow \max , F(x, y) \geq \alpha, x \in X, y=\left(y_{1}, \ldots, y_{l}\right) \in Y .
$$

Then a fuzzy set with the membership function of the form

$$
\mu_{\alpha}(x)= \begin{cases}\mu_{\tilde{X}}(x), & x \in P_{\alpha}(F, \tilde{X}), \\ 0, & x \notin P_{\alpha}(F, \tilde{X})\end{cases}
$$

is called the solution of the vector fuzzy optimization problem with the fuzzy set of alternatives and the degree of nondominatedness of alternatives no less than $\alpha$. 
Thus, the fuzzy set of solutions of the original problem contains those and only those alternatives with the degree of nondominatedness no less than $\alpha$ that are efficient with respect to both estimates of alternatives $y_{i}, i \in N_{l}$, and the function $\mu_{\tilde{X}}(x)$ of the membership in the fuzzy set of alternatives $\tilde{X}$. A specific alternative is chosen out of them by multicriteria optimization methods. Moreover, the maximization of a vector objective function may be replaced with attaining a preassigned value of the vector criterion that corresponds to sufficing the initial objective.

\section{SOME APPROACHES TO SOLVING THE PROBLEM}

There are many methods to solve multicriteria problems; however, most of them are intended to solve problems of the choice of alternatives in nonfuzzy environments. A modification makes them applicable under fuzzy conditions as well.

Developing methods to solve the problem $Z(F, X)$ under fuzziness needs operations of finding the sum, product, minimum, and maximum of fuzzy quantities to be known and their results to be used.

By a fuzzy number we will mean a fuzzy set with the domain of definition as an interval of the real axis $R$.

Denote the set of all the fuzzy numbers defined on $R^{1}$ by $\widetilde{R}^{1}$. Let $x$ and $y$ be two fuzzy numbers with the supports $S_{x}=\left(a_{1}, a_{2}\right)$ and $S_{y}=\left(b_{1}, b_{2}\right)$, respectively; $a_{2}>a_{1}, b_{2}>b_{1}$; and $g: R^{1} \times R^{1} \rightarrow R^{1}$ be some function. Then by the generalization principle [12], the fuzzy number $D=g(x, y)$ is defined by the membership function

$$
\mu_{D}(z)=\sup _{\substack{g(a, b)=z \\ a \in S_{x}, b \in S_{y}}} \min \left\{\mu_{x}(a), \mu_{y}(b)\right\} .
$$

Let $\otimes$ be one of the four arithmetic operations:,,$+- \cdot$, and $/ ; g(a, b)=a \otimes b$. Then formula (4) determines the result of the arithmetic operation $\otimes$ over the fuzzy numbers $x$ and $y$. If $g(\cdot)$ is a function of not two but $n$ arguments, the generalization principle is formulated similarly to (4).

When comparing two fuzzy quantities, it is necessary to define the equality of these quantities.

Definition 5. Two fuzzy quantities (two numbers) $\left(x_{1}, \mu_{1}\left(x_{1}\right)\right)$ and $\left(x_{2}, \mu_{2}\left(x_{2}\right)\right)$ are assumed equal if $x_{1}=x_{2}$ and $\mu_{1}\left(x_{1}\right)=\mu_{2}\left(x_{2}\right)$.

Definition 6. If the conditions $x_{1} \geq x_{2}$ and $\mu_{1}\left(x_{1}\right) \geq \mu_{2}\left(x_{2}\right)$ are satisfied and one of these inequalities is strict, then the fuzzy quantity $\left(x_{1}, \mu_{1}\left(x_{1}\right)\right)$ is greater than the fuzzy quantity $\left(x_{2}, \mu_{2}\left(x_{2}\right)\right)$.

An approach based on the method of successive concessions is developed. In solving a multicriteria problem by the successive concession method, first, the qualitative analysis of the relative importance of partial criteria is carried out. Criteria of the problem are enumerated in advance in the order of decreasing importance; thus, $f_{1}(x)$ is the most important criterion, $f_{2}(x)$ is less important, they are followed by other partial criteria: $f_{3}(x), f_{4}(x), \ldots, f_{l}(x)$. The most important criterion $f_{1}(x)$ is maximized and its greatest value $f_{1}^{*}$ is found. Then an admissible decrease (concession) $\Delta_{1} \geq 0$ of the criterion $f_{1}(x)$ is specified and the greatest value $f_{2}^{*}$ of the second criterion $f_{2}(x)$ is found provided that the value of the first criterion should be no less than $f_{1}^{*}-\Delta_{1}$. Then a concession $\Delta_{2} \geq 0$ is specified for the second criterion, which together with the first concession is used to find the conditional maximum of the third criterion, etc. Finally, the least important criterion $f_{l}(x)$ is maximized provided that the value of each of the $l-1$ previous criteria $f_{r}(x)$ is no less than $f_{r}^{*}-\Delta_{r}$, then the obtained alternatives are considered to be optimal.

Thus, the solution method is implemented by executing a multistage procedure and sequentially includes the constraints of the problem $Z(F, X)$ and allows for the structural features of its feasible domain. The optimal solution is that of the last problem in the sequence of problems

$$
\begin{gathered}
f_{1}^{*}=\max \left\{f_{1}(x) \mid x \in X\right\}, f_{2}^{*}=\max \left\{f_{2}(x) \mid x \in X, f_{1}(x) \geq f_{1}^{*}-\Delta_{1}\right\}, \\
\ldots, f_{l}^{*}=\max \left\{f_{l}(x) \mid x \in X, f_{r-1}(x) \geq f_{r-1}^{*}-\Delta_{r-1}, r \in N_{l} \backslash\{1\}\right\} .
\end{gathered}
$$

Note that if all the $\Delta_{r}$ are zero, the method of successive concessions separates out only lexicographically optimal solutions that provide the greatest value on the set of feasible solutions to the most important criterion $f_{1}(x)$. Therefore, the 
values of concessions intended to solve a multicriteria problem can be considered as a measure of the deviation of the priority (the degree of relative importance) of partial criteria from the rigid, lexicographic one.

The concepts of domination structures and nondominated solutions in multicriteria problems allow considering general cases where the information about DM's preferences is available. The paper [17] introduces the concepts of fuzzy convex and fuzzy polar cones, which generalize the structures used to define the concepts of Pareto, Slater, Smale, etc. optimality.

If there is no information on the preferences both on the set of alternatives and on the set of criteria, elementary methods such as minimax, maximax, etc. are usually used. Given the information only about the comparative importance of estimates with respect to each criterion, alternatives are considered successively with respect to individual criteria (lexicographic method, permutation method, successive reduction of residuals, etc.). If DM's preferences on the set of criteria estimates are expressed in ordinal scales and specified with respect to the weights of the criteria, voting methods are used, among which Roy's method is best known in decision making.

If it is possible to obtain relative weights of criteria and relative values of criteria estimates with respect to individual criteria, many various methods can be applied such as direct estimation of alternatives with the use of preset estimating functions (for example, additive weighted convolution of estimates over all the criteria) and methods of the utility theory, which require continuous dialogue with the DM.

If perfect criteria estimates are known along with criteria importance, methods of the estimate of the attainability of objectives can be applied. These methods are detailed in [10, 18].

Let us consider some simple methods of the choice of alternatives in the lack of the information on preferences on the set of criteria [12] and show how the domain of their application can be extended.

Let fuzzy estimates $f_{i j}=f_{i}\left(x_{j}\right)$ of alternatives $x_{j}, j \in N_{n}$, with respect to criteria $f_{i}, i \in N_{l}$, be given or calculated. To calculate fuzzy estimates one can use Statement 2 from [6].

\section{METHOD OF SEARCHING FOR LEXICOGRAPHICALLY OPTIMAL SOLUTIONS OVER A FUZZY FEASIBLE COMBINATORIAL SET OF PERMUTATIONS}

Applying the method to problems with fuzzy initial information reduces to the following steps.

1. Order the criteria according to the importance: $f_{1}(x), f_{2}(x), \ldots, f_{l}(x)$.

2. Specify a level (agreed with the DM) $\alpha \in[0,1]$ for which the set of the best alternatives is found according to steps $3-5$.

3. Determine the lower $(l)$ and upper $(u)$ bounds of $\alpha$-level subsets for the estimate of alternatives according to the considered criterion:

$$
l\left(f_{i j}\right)=\inf _{\mu_{f_{i j}}(x) \geq \alpha}, \quad u\left(f_{i j}\right)=\sup _{\mu_{f_{i j}}(x) \geq \alpha} .
$$

4. For each pair of alternatives $z, y \in X$ calculate the parameters of the mutual excess of the criteria estimates $\Delta_{z y}(z<y)$ and $\Delta_{y z}(y<z)$ :

(a) if the estimates are such that the inclusion $f_{i y}^{\alpha} \subset f_{i z}^{\alpha}$ holds, then

$$
\Delta_{z y}=\frac{u\left(f_{i z}\right)-u\left(f_{i y}\right)}{u\left(f_{i z}\right)-l\left(f_{i z}\right)}, \Delta_{y z}=\frac{l\left(f_{i y}\right)-l\left(f_{i z}\right)}{u\left(f_{i z}\right)-l\left(f_{i z}\right)},
$$

where $z, y \in A$;

(b) if the estimates intersect and $\exists x_{0} \in S_{f_{i z}}: \forall y \in S_{f_{i y}} x_{0}>y$, then

$$
\Delta_{z y}=1-\frac{u\left(f_{i y}\right)-l\left(f_{i z}\right)}{\max \{r(z), r(y)\}}, \Delta_{y z}=0,
$$

where $r(z)=u\left(f_{i z}\right)-l\left(f_{i z}\right)$ and $r(y)=u\left(f_{i y}\right)-l\left(f_{i y}\right)$;

(c) If the estimates do not intersect and $\forall x \in S_{f_{i z}}, \forall y \in S_{f_{i y}} x>y$, then

$$
\Delta_{z y}=1, \Delta_{y z}=0 \text {. }
$$


5. Calculate the parameters $\mu_{D_{i j}}$ of the membership of the $j$ th alternative in the set of the best ( $D$-set) alternatives with respect to the $i$ th criterion

$$
\mu_{D_{i j}}=\max \left\{0,\left(\max _{j \in X} \Delta_{j y}-\max _{\substack{y \in X \\ y \neq j}} \Delta_{y j}\right)\right\},
$$

where $\Delta_{j y}$ and $\Delta_{y j}$ are calculated by the formulas (5) and (6) for the $i$ th criterion.

6. If the $D$-set with respect to the considered criterion contains one alternative with $\mu_{D_{i j}} \geq \alpha$, then consider it the best. If the $D$-set contains more than one alternative with $\mu_{D_{i j}} \geq \alpha$, then choose the criterion with the next degree of importance and repeat steps 3-5. If all the criteria are tested, the $D$-set contains more than one alternative, and $\alpha<1$, then increase $\alpha$ and go to Step 3. If $\alpha=1$, then the DM makes the final choice of the best alternative.

Consider the choice of alternatives according to a generalized maximin criterion, which is a development of the Wald method for fuzzy alternatives.

\section{METHOD OF THE CHOICE OF ALTERNATIVES BY A GENERALIZED MAXIMIN CRITERION}

The method includes the following steps.

1. For each criterion, calculate the fuzzy maximum criterion estimate $f_{i \max }=\max \left\{f_{i}\left(x_{j}\right) \mid x_{j} \in X, j \in N_{n}\right\}, i \in N_{l}$.

2. Calculate the reduced normalized estimates of alternatives according to the criteria $f_{i j}{ }^{*}=f_{i}\left(x_{j}\right) / f_{i \max }$, $x_{j} \in X, j \in N_{n}$.

3. Calculate the minimum criterion estimate for each alternative $f_{j \min }$ defined as $f_{j \min }=\tilde{\min }\left\{f_{i j}{ }^{*} \mid i \in N_{l}\right\}$, $j \in N_{n}$.

4. Find the generalized maximum of the obtained minimum estimates $f_{0 \max }=\max \left\{f_{j \min } \mid j \in N_{n}\right\}$.

5. Evaluate the degree of similarity $f_{0 \text { max }}$ of each of the estimates $f_{j \min }$. As a parameter of similarity of fuzzy numbers, $\xi_{j}=\sum_{z \in[0,1]}\left|\mu_{f_{0 \max }}(z)-\mu_{f_{\text {min }}}(z)\right|$ can be used.

6. Choose the alternative with the maximum index $\xi_{j}$.

If the alternative is chosen according to the maximax principle, calculate $f_{j \max }=\max \left\{f_{i j}{ }^{*} \mid i \in N_{l}\right\}, j \in N_{n}$, instead of $f_{j \min }$ in Item 3 and in other sections and use the value $f_{j \max }, j \in N_{n}$, instead of $f_{j \min }$.

\section{MATHEMATICAL MODELS OF SOME APPLICATION PROBLEMS}

Two-Criteria Traveling Salesman Problem. The traveling salesman problem is a classical discrete optimization problem [3]. It consists in compiling a route of a trading agent (being at an initial point) to visit $(n-1)$ localities. The cost of moving from one locality to another is known and specified by a matrix $C=\left[c_{i j}\right]_{n \times n}$. Only a route that provides visiting once all the localities $n$ and returning to the initial point is feasible. The best route should obviously minimize the total cost of relocation.

Feasible solutions in this problem are connected routes, which are uniquely defined by the ordered set of localities visited by the salesman. Each such route can be identified with the permutation $p=\left(i_{1}, i_{2}, \ldots, i_{n}\right)$ of $n$ numbers (ordered sample of $n$ numbers out of a set of $n$ numbers).

Thus, the mathematical statement of the traveling salesman problem has the form

$$
\min \left\{f(p)=\sum_{k=1}^{n} c_{i_{k} i_{k+1}} \mid p=\left(i_{1}, i_{2}, \ldots, i_{n}\right) \in P\right\},
$$

where $P$ is the set of permutations of numbers from 1 to $n$. 
Note that for an arbitrary (even symmetric) matrix $C$ the traveling salesman problem is $N P$-complete. It has many analogs.

In particular, such a model results from the problem of scheduling the readjustment of the equipment that can be used to manufacture different types of products but requires certain (time or material) expenditures when passing from one operating conditions to others. Obviously, the traveling salesman problem can be generalized to the case of many criteria and fuzzy set of alternatives.

Let $x=\left(i_{1}, i_{2}, \ldots, i_{n}\right)$, being an arbitrary fuzzy permutation of elements of the set $N_{n}=\{1,2, \ldots, n\}$ of locality numbers, be a route of the traveling salesman. Let us associate each route $x$ with two criteria:

$$
\begin{gathered}
f_{1}(x)=\sum_{k=1}^{n} c_{i_{k} i_{k+1}}, \\
f_{2}(x)=\max _{\left(i_{k}, i_{k+1}\right)} c_{i_{k} i_{k+1}}, k=1,2, \ldots, n,
\end{gathered}
$$

where $i_{n+1}=i_{1}$. Criterion $f_{1}(x)$ is the length of the route $x$ and $f_{2}(x)$ is the bottleneck of this route. A problem with one criterion (8) is called linear, and a problem with criterion (9) is a bottleneck problem.

Let $F(x)=\left(f_{1}(x), f_{2}(x)\right)$. The task is to minimize the vector criterion $F(x)$ over a fuzzy set $\tilde{X}: \min \{F(x) \mid x \in \tilde{X}\}$.

Bank Lending. As market relations develop, the process of granting credits to enterprises is known to involve numerous risk factors, which may cause outstanding payments in due time. The analysis of the credited party reveals the capability of complete debt redemption in due time and loan debt extinguishment; the degree of the risk that the bank is ready to undertake; the amount of credit to be granted in a certain situation; and credit terms.

In modern conditions, credit analysis [19] involves not only the assessment of the client's solvency at specified date but also revealing the most preferable loan debtors, predicting their financial stability in the long term, and allowing for possible risks related to credit operations. Such a comprehensive analysis allows a bank to administer credit resources more efficiently and to obtain a profit.

The methods applied by banks in the field of lending are based on accounting reports; therefore, they only allow assessing the credit status of the borrower without providing a choice of the most optimal borrowers in order to minimize risk factors for the bank and to efficiently plan the activity.

Let us set up a mathematical model based the theory of fuzzy sets and multicriteria optimization, which allows increasing the validity of decisions made in the field of lending and provides the choice of the most rational alternatives from the set of feasible ones. The problem of resource allocation among the alternatives is of current importance. For example, of interest are combinatorial optimization problems of determining the combinations of alternatives (projects) that maximize the efficiency (or the efficiency per unit resource) and satisfy certain resource constraints.

Assume that enterprises applied to some bank for a loan. Since resources of the bank are limited, there is a problem of choosing enterprises that are the best in a complex of performance criteria. In the problem under study, enterprises are the alternatives $a_{1}, \ldots, a_{n}$, among which the best ones should be chosen. To assess the credit status of the borrower enterprises, data of their accounting reporting are used: cash assets $\left(c_{1}\right)$, short-term financial investments $\left(c_{2}\right)$, accounts receivable $\left(c_{3}\right)$, stock and expenses $\left(c_{4}\right)$, owned capital $\left(c_{5}\right)$, short-term liabilities $\left(c_{6}\right)$, balance sheet total $\left(c_{7}\right)$, gross proceeds $\left(c_{8}\right)$, and profit $\left(c_{9}\right)$, used to calculate the coefficients that characterize the credit status of borrowers: cash ratio $\left(F_{1}\right)$, intermediate coverage ratio $\left(F_{2}\right)$, total coverage ratio $\left(F_{3}\right)$, equity ratio $\left(F_{4}\right)$, and cost of goods percentage $\left(F_{5}\right)$. These factors are the performance criteria of the credit status of the enterprises and can be calculated by the following formulas [19]:

$$
F_{1}=\frac{c_{1}+c_{2}}{c_{6}}, F_{2}=\frac{c_{1}+c_{2}+c_{3}}{c_{6}}, \quad F_{3}=\frac{c_{1}+c_{2}+c_{3}+c_{4}}{c_{6}}, F_{4}=\frac{c_{5}}{c_{7}}, F_{5}=\frac{c_{9}}{c_{8}} .
$$

To develop a mathematical model, let us consider a fuzzy set $\widetilde{A}=\left\{a_{1}, \mu_{\widetilde{A}}\left(a_{1}\right), a_{2}, \mu_{\widetilde{A}}\left(a_{2}\right), \ldots, a_{q}, \mu_{\widetilde{A}}\left(a_{q}\right)\right\}$ consisting of unities and zeroes. Consider a set of combinations $C(\widetilde{A})$ of elements $a=\left(a_{i_{1}}, \mu_{\widetilde{A}}\left(a_{i_{1}}\right), a_{i_{2}}, \mu_{\widetilde{A}}\left(a_{i_{2}}\right)\right.$, $\left.\ldots, a_{i_{n}}, \mu_{\widetilde{A}}\left(a_{i_{n}}\right)\right)$ from the fuzzy set $\tilde{A}$, where $a_{i_{j}} \in \tilde{A} \forall i_{j}, j \in N_{k}, i_{s} \neq i_{t}$, if $s \neq t \forall s \in N_{k}, \forall t \in N_{k}$. Introduce a variable

$$
x_{i}= \begin{cases}1 & \text { if the } i \text { th enterprise is chosen to be granted a credit } \\ 0 & \text { otherwize. }\end{cases}
$$


The mathematical model of the problem has the form

$$
\max \{F(x) \mid x \in \tilde{X}\},
$$

where $F(x)=\left(F_{1}(x), F_{2}(x), \ldots, F_{5}(x)\right)$ and $\tilde{X}$ is a fuzzy set of combinations of alternatives.

With the use of the mathematical toolkit of fuzzy set theory, the problem is solved in three stages.

1. Membership functions that correspond to the concepts of coefficients introduced above, which characterize the credit status of the borrowers, are constructed by experts in lending to various types of enterprises.

2. Specific values of membership functions according to performance criteria $F_{1}, \ldots, F_{5}$, which correspond to the considered alternatives are determined.

3. The proposed methods are applied in order to reveal an alternative that is the best according to all the criteria. Optimal are the alternatives that maximize the vector criterion and have the maximum value of the function of membership in the set $X$. If the criteria used to choose the alternatives are of identical importance, the generalized maximum criterion is used.

If the criteria are ordered in importance, the method of searching for lexicographic solutions is applied.

\section{CONCLUSIONS}

We have analyzed vector problems over fuzzy combinatorial sets of alternatives, developed methods to solve them, and presented mathematical models of some application problems over combinatorial sets of permutations and combinations in case of fuzzy initial information.

Based on the information about the convex hull of the feasible domain and on the properties of the polyhedron whose vertices define a fuzzy combinatorial set of permutations, a method to solve complex multicriteria problems over this combinatorial set has been developed and justified. The use of the structural properties of combinatorial polyhedra allows creating efficient solution algorithms for new classes of vector combinatorial optimization problems with fuzzy data. Other multicriteria choice methods modified for the case of fuzzy information can be applied depending on the specific features of the problem. Generalizing an exact method does not involve difficulties if the ways of representing fuzzy concepts, implementing fuzzy computations, comparing fuzzy numbers, setting up a fuzzy set of the best alternatives are adequate to the conditions of the problem being solved.

\section{REFERENCES}

1. L. A. Zadeh, "Fuzzy sets," Inform. and Control, 8, 338-353 (1965).

2. V. V. Podinovskii and V. D. Nogin, Pareto-Optimal Solutions of Multicriteria Problems [in Russian], Nauka, Moscow (1982).

3. I. V. Sergienko and V. P. Shilo, Discrete Optimization Problems: Challenges, Solution Techniques, and Investigations [in Russian], Naukova Dumka, Kyiv (2003).

4. N. V. Semenova and L. N. Kolechkina, Vector Discrete Optimization Problems Over Combinatorial Sets: Methods of the Analysis and Solution, [in Ukrainian], Naukova Dumka, Kyiv (2009).

5. T. T. Lebedeva, N. V. Semenova, and T. I. Sergienko, "Optimality and solvability conditions in linear vector optimization problems with convex feasible sets," Dop. NAN Ukrainy, No. 10, 80-85 (2003).

6. N. V. Semenova, L. N. Kolechkina, and A. N. Nagirna, "An approach to solving discrete vector optimization problems over a combinatorial set of permutations," Cybern. Syst. Analysis, 44, No. 3, 441-451 (2008).

7. N. V. Semenova, L. N. Kolechkina, and A. N. Nagornaya, "Solution and investigation of vector problems of combinatorial optimization on a set of polypermutations," J. Autom. Inform. Sci., Vol. 40, Issue 12, 27-42 (2008).

8. N. V. Semenova, "Optimality conditions in vector combinatorial optimization problems," Teoriya Optim. Rishen', No. 7, 153-160 (2008).

9. N. V. Semenova, L. N. Kolechkina, and A. M. Nagirna, "Solving multicriteria optimization problems over a set of polypermutations," Dop. NAN Ukrainy, No. 2, 41-48 (2009). 
10. A. N. Averkin, I. Z. Batyrshin, A. F. Blishun, et al., D. A. Pospelov (ed.), Fuzzy Sets in Models of Control and Artificial Intelligence [in Russian], Nauka, Moscow (1986).

11. S. A. Orlovskii, Problems of Decision Making under Fuzzy Initial Information [in Russian], Nauka, Moscow (1981).

12. A. N. Borisov, A. V. Alekseev, G. V. Merkur'eva, et al., Processing Fuzzy Information in Decision Making Systems [in Russian], Radio i Svyaz', Moscow (1989).

13. I. V. Sergienko and M. F. Kaspshitskaya, Models and Methods for the Computer Solution of Combinatorial Optimization Problems [in Russian], Naukova Dumka, Kyiv (1981).

14. V. A. Emelichev, M. M. Kovalev, and M. K. Kravtsov, Polyhedra, Graphs, and Optimization [in Russian], Nauka, Moscow (1981).

15. Yu. G. Stoyan and S. V. Yakovlev, Mathematical Models and Optimization Methods of Geometric Design [in Russian], Naukova Dumka, Kyiv (1986).

16. O. O. Emets and O. O. Emets, "Some operations and relations over fuzzy numbers," Nauk. Visti NTUU "KPI," No. 5, 39-46 (2008).

17. E. Takeda and T. Nishida, "Multiple criteria decision problems with fuzzy domination structures," Fuzzy Sets and Syst., 3, 123-136 (1980).

18. E. Jaquet Lagreze, "Applying fuzzy relations to estimate the preferability of distributed quantities," in: Statistical Models and Multicriteria Decision-Making Problems [in Russian], Statistika, Moscow (1979), pp. 168-183.

19. A. V. Andreichikov and O. N. Andreichikova, Analysis, Synthesis, and Planning of Economic Decisions [in Russian], Finansy i Statistika, Moscow (2001). 